Baltic Astronomy, vol. 9, 247-252,2000.

\title{
AN IUE ARCHIVAL STUDY OF THE WHITE DWARF TEMPERATURE VARIATION IN THE DWARF NOVA WZ SAGITTAE
}

\author{
R. J. Slevinsky, D. Stys, S. West, E. M. Sion and F. H. Cheng \\ Department of Astronomy and Astrophysics, Villanova University, U.S.A.
}

Received January 15, 2000

\begin{abstract}
The IUE archive offers a series of far UV spectra of ultrashort period dwarf nova WZ Sge over a long time baseline and hence the opportunity to monitor the ongoing accretion, the cooling of the white dwarf and temporal variations in the line spectra. We have carried out a quantitative analysis of these spectra including model atmosphere simulations. We find an indicated cooling of the white dwarf by $5140 \mathrm{~K}$ from $20510 \mathrm{~K}$ to $15370 \mathrm{~K}$ with a thermal e-folding time of 690 days. This cooling rate is well-represented by heating of the white dwarf by accretion of matter with angular momentum during the December 1978 outburst followed by subsequent cooling. We find that the abundance of carbon in the white dwarf photosphere is elevated above solar. This finding is consistent with the results of recent HST observations. We find marginal evidence that the $\mathrm{C}$ abundance was $6 \mathrm{x}$ solar, close to the outburst and $2 \mathrm{x}$ solar in the most recent spectra obtained 13.7 years later.
\end{abstract}

\section{Key words:}

\section{INTRODUCTION}

The dwarf nova, WZ Sge, has an ultra-short orbital period (81 minutes, 38 seconds), one of the largest outburst amplitudes ( 7 magnitudes), and the longest recurrence time (33 years, the last occurred on 1978 December 1-2) of any dwarf novae. This short orbital period makes WZ Sge a focus for the study of gravitational wave emission and its role in mass transfer. A coherent 28 second signal is detectable in the optical (Robinson, Nather \& Patterson 1978, hereafter 
RNP; Patterson 1980, Skidmore et al.1997), the ultraviolet (Welsh et al.1997), and soft X-ray wavelengths, due most likely to nonradial pulsation (RNP 1978) or to the rotation of the white dwarf (Patterson 1980), but the actual origin of the pulsation is still in question (see however Cheng et al. 1997). If the 28 second signal is due to pulsation, then the role of accretion and its associated heating must be explored. Warner (1998) has raised the possibility that accretion, by changing the white dwarf surface temperature, may influence whether the white dwarf lies within or outside the ZZ Ceti instability strip.

We had previously carried out temperature and chemical abundance analyses of spectra obtained with the Hubble Space Telescope Goddard High-Resolution Spectrograph using the G140L grating, and with the FOS and $\mathrm{G} 130 \mathrm{H}$ grating. All spectra are dominated by strong, broadened photospheric C I absorption features centered at 1356 and 1464 and N I centered at 1494. The spectra also show Stark-broadened Ly $\alpha$ red absorption with, $\mathrm{H}_{2}$ quasi-molecular Ly $\alpha$ "satellite" absorption lines, and Si IV 1393, 1402 absorption lines. Our best fitting synthetic spectra yielded a rapidly rotating white dwarf with velocity $V_{\text {rot }} \sin i=1200_{-400}^{+300} \mathrm{~km} \mathrm{~s}^{-1}$ (Cheng et al. 1997), white dwarf effective temperature $T_{\mathrm{wd}}=14800 \mathrm{~K}$, the gravity log $\mathrm{g}=8.0$, the chemical abundances with $3 \sigma$ error-bars, in number relative to solar, C, $5.0_{-2.0}^{+2.0} ; \mathrm{N}, 3.0_{-1.0}^{+1.0} ; \mathrm{Si},<0.1$; and all other metals, 0.01. Earlier FOS $\mathrm{G} 130 \mathrm{H}$ spectra reported by Sion and coworkers reveal rotational velocity $V_{\mathrm{rot}} \sin i=1100_{-400}^{+400} \mathrm{~km} \mathrm{~s}^{-1}, T_{\mathrm{wd}}=14800$ $\mathrm{K}$, the gravity $\log g=8.3$ the chemical abundances, C, $2.0_{-1.5}^{+5.0} ; \mathrm{N}$, $1.0_{-1.0}^{+3.0} ; \mathrm{Si},<0.1$; all other metals, 0.01 .

Following the IUE identification of dominant C I, Si I and Si II in the atmosphere of WZ Sge (Sion et al. 1990), and the followup single epoch HST FOS and GHRS G140L synthetic spectral analyses by Sion et al. (1995) and Cheng et al. (1997), the need for further information on the variations of the white dwarf temperature and the peculiar line spectrum has heightened. In particular, a more quantitative characterization of the white dwarf cooling since the December 1978 outburst is needed. The IUE archive offers a series of far UV spectra over a long time baseline and hence the opportunity to monitor the ongoing accretion, the cooling of the white dwarf and temporal variations in the line spectra. We have analyzed twelve archival spectra from the International Ultraviolet Explorer (IUE), in an attempt to shed further light on this topic. 


\section{IUE FAR ULTRAVIOLET SPECTROSCOPIC DATA}

Twelve large aperture, low dispersion IUE images were taken of the dwarf nova WZ Sagittae between November 1981 and August 1992 with the Short Wavelength Prime (SWP) camera, covering the wavelength range $1160-2000 \AA$. These spectra were obtained throughout the quiescent period following the 1978 outburst, the last image taken in 1992 August. Exposure times ranged from 90 to 295 minutes, and the images were reduced using the standard NEWSIPS reductions provided by NASA/Goddard. The image numbers were SWP 5761, 8827, 10909, 15554, 29589, 31294, 33419, 36259, 36885, 45103, 45298, 45398 and 45370.

The earliest spectrum is SWP 5761 and the latest spectrum is SWP 45370. All of the spectra show the broadened Lyman- $\alpha$ absorption profile, the $\mathrm{H} 2$ quasi-molecular absorption Lyman- $\alpha$ satellite line characteristic of the high gravity of the white dwarf and a rich array of absorption features dominated by $\mathrm{C}$ I resonance lines including the strongest CI feature at $1657 \AA$, as well Si II and Si III absorption.

Emission features are also present (besides the geocoronal Lyman $\alpha$ feature) including C IV 1550 due to the contribution of the accretion disk structure in quiescence. The CIV emission line remains fairly constant in width and flux over the time interval covered by the later spectra (SWP 45103, SWP 45298 and SWP 45370). Si III emission persists throughout the series of spectra. Over all $12 \mathrm{spec}-$ tra, however, there are variations in both the number of emission features and the individual emission strengths. These variations in emission are almost certainly tied to variations in accretion in the system.

Another noticeable trend throughout the 12 spectra is the variation of the flux level. Since its outburst in 1978 December, the white dwarf in WZ Sge has exhibited overall cooling trends. The first image (SWP 05761), taken in 1979, has an elevated flux level in comparison to the remainder of the spectra, due simply to its proximity to the outburst event. The next spectrum reveals a white dwarf that has cooled significantly in comparison to the previous, but the following spectra reveal higher flux levels, indicating higher temperature. The "warming" of the white dwarf must be due to the ongoing accretion in the system. Changes in the flux level reflect variations in temperature based on the amount of material being accreted. 


\section{SYNTHETIC SPECTRAL ANALYSIS}

Using the codes TLUSTY and SYNSPEC (Hubeny 1988, Hubeny \& Lanz 1995), we generated model atmospheres to obtain abundances, temperature, and surface gravity of the underlying white dwarf. The value of $V \sin i$ was kept fixed at $1200 \mathrm{~km} / \mathrm{s}$ (Cheng et al. 1997) during the fitting procedure. The L $\alpha$ and CIV emission regions have been masked in our procedure.

In order to attempt a more refined fit to individual spectra, we carried out the following experiment. Given the relatively poor signal to noise of the IUE spectra and uncertainties associated with the synthetic spectra, a detailed comparison with best fit model parameters corresponding to very similar spectra closely spaced in time would not yield definitive results. Instead we have selected a few spectra, three of them (SWP 5761, SWP 8827, SWP 10909) close in time to the outburst and a second pair of spectra (SWP 45103, SWP 45370) as far into quiescence as IUE covered, in order to search for any chemical abundance and temperature differences over the longest time baseline. For this experiment we generated a grid of new models with $\mathrm{L} \alpha$ satellite hydrogen absorption and used the routine GRID FIT to get more accurate white dwarf abundances and temperatures.

The earlier spectra close to the outburst look quite different from the late spectra. For example, SWP8827 and SWP10909 look quite different despite being fit with models having similar parameters. This would imply that the spectral variations seen from early to late quiescence cannot be ascribed simply to a cooling of the white dwarf. We suggest that variations in the rate of accretion and possibly the contribution of a hot accretion belt (or inhomogeneous temperature variation across the white dwarf surface) complicate the simple single temperature cooling picture.

The earlier spectra also show an excess in continuum flux in the region 1250-1500 $\AA$. We tried models with higher $T_{\text {eff }}$ to improve the continuum fit but found that the fit to the $L \alpha$ profile became worse. We also tried photosphere plus absorbing curtain fits but the resulting best fit barely differs from a fit with a single $T_{\text {eff }}$ and no curtain.

Since the IUE data includes no error-bars, we adopted the following procedure. We took the error dispersion as

$$
\sigma_{\lambda}=0.1 \times F_{\lambda}+\left[5 \times 10^{-15}\right]
$$


This error-bar is taken to be $10 \%$ of the flux, but we added a small quantity of flux, $5 \times 10^{-15}$, to avoid assigning too much weight to the low flux levels. The best-fit model to each spectrum gave the reduced $\chi^{2}$ values listed in table 1 , where the results of the fitting are presented.

Table 1.

\begin{tabular}{lrrrrrc}
\hline SWP spectrum & $\log g$ & $T_{\text {wd }}$ & Si & C & $V_{\text {rot }}$ & $\frac{\chi^{2}}{495}$ \\
\hline Early spectra & & & & & & \\
SWP 05761 & 8 & 20510 & 0.1 & 6.0 & 1200 & $4.88 \times 10^{-2}$ \\
SWP 08827 & 8 & 17730 & 0.1 & 6.0 & 1200 & $5.38 \times 10^{-2}$ \\
SWP 10909 & 8 & 17300 & 0.1 & 6.0 & 1200 & $4.70 \times 10^{-2}$ \\
Late spectra & & & & & & \\
SWP 45103 & 8 & 15310 & 0.1 & 2.0 & 1200 & $3.51 \times 10^{-2}$ \\
SWP 45370 & 8 & 15370 & 0.1 & 2.0 & 1200 & $3.44 \times 10^{-2}$ \\
\hline
\end{tabular}

As seen in Table 1, the white dwarf cooled from a maximum of $20510 \mathrm{~K}$ three months after the return to quiescence, down to 15300 $\mathrm{K}$ in August 1992, 13.7 years after the outburst. Despite the noisy spectra, there is marginal evidence from fitting the C I and C II lines that the photospheric carbon abundance has changed between the value determined in the early spectra of $6 \mathrm{x}$ solar, down to a carbon abundance of $2 x$ solar, in the most recent pair of spectra. However, we are forced to conclude that our evidence of a $\mathrm{C}$ abundance change is marginal at best due to the lack of sufficient spectral quality.

\section{DISCUSSION}

Our results have indicated a cooling of the white dwarf by $5000 \mathrm{~K}$ over a time baseline of 13.7 years. The e-folding time of this cooling is 687 days (Sion 1999). Since the earliest quiescent IUE spectrum was secured 3 months after the return to optical quiescence following the December 1978 outburst, this amount of cooling is a lower limit. The highest $T_{\text {eff }}$ measured for the white dwarf is $20510 \mathrm{~K}$, at 0.25 years post-outburst. A range of $T_{\text {eff }}$ similar to this was estimated by LaDous (1993) based upon comparing template IUE SWP spectra of single DA white dwarfs with the IUE spectra of WZ Sge. The most recent archival IUE spectrum yielded $T_{\text {eff }}=15370 \mathrm{~K}$. Sparks et al. (1993) have shown that the released energy associated with shear 
mixing of the accreted matter accounts for the cooling of the white dwarf whereas radial accretion produces a too rapid cooling.

There is no question that the $\mathrm{C}$ abundance is enhanced relative to all other elements in WZ Sge's white dwarf. Explaining the overabundance of $\mathrm{C}$ poses a vexing problem. Carbon abundance we derive from the CI absorption features in the IUE spectra is comparable to the HST-derived abundances, the accretion rate derived by Sion et al. (1995) remains roughly valid for the case of the IUEderived abundances.

ACKNOWLEDGMENTS. This work was supported by NASA HST grant AR7300 and NASA ADP grant NAG 5-8388 to Villanova University. Three of us (R.S., D.S., S.W.) were supported by summer undergraduate research assistantships from the NASA Delaware Space Grant Colleges Consortium.

\section{REFERENCES}

Cheng F., Sion E. M., Szkody P., Huang M. 1997, ApJ, 484, L149

Hubeny I. 1988, Comput. Phys. Comm., 52, 103

Hubeny I., Lanz T. 1995, ApJ, 439, 875

LaDous C. 1993, unpublished

Patterson J. 1980, ApJ, 241, 235

Robinson E. L., Nather R.E., Patterson J. 1978, ApJ, 219, 168 (RNP)

Sion E. M. 1999, PASP, in press

Sion E. M., Leckenby H., Szkody P. 1990, ApJ, 364, L41

Sion E. M., Szkody P., Cheng F.H., Huang, M. 1995b, ApJ, 444, L97

Skidmore W., Welsh W. F., Wood J. H., Stiening R. F. 1997, MNRAS, 288, 198

Sparks W.M., Sion E. M., Starrfield S. G., Austin S. 1993, Annals of the Israel Physical Society, 10, 96

Welsh W.F., Skidmore W., Wood J.H., Cheng F.H., Sion E. M. 1997, MNRAS, 291, 57 